\title{
Factores predictores de reingreso hospitalario no planificado en pacientes dados de alta de una Unidad de Corta Estancia Médica
}

\author{
M.T. DIZ-LOIS PALOMARES, F. DE LA IGLESIA MARTÍNEZ, R. NICOLÁS \\ MIGUEL, C. PELLICER VÁZQUEZ, V. RAMOS POLLEDO, F. DIZ-LOIS MARTÍNEZ \\ Unidad de Corta Estancia Médica. Complexo Hospitalario Juan Canalejo. A Coruña.
}

FACTORS THAT PREDICT UNPLANNED HOSPITAL READMISSION OF PATIENTS DISCHARGED FROM A SHORT STAY MEDICAL UNIT

\section{RESUMEN}

Objetivo: Determinar los factores independientes que predicen el reingreso hospitalario no planificado de pacientes que ingresan en una Unidad de Corta Estancia Médica (UCEM).

Diseño y ámbito del estudio: Cohorte prospectiva. Area sanitaria: 500000 habitantes.

Sujetos: Pacientes que sobrevivieron a un ingreso hospitalario en la $\operatorname{UCEM}(\mathrm{n}=196, \mathrm{a}=0,05, \mathrm{~d}=7$ ).

Mediciones o intervenciones: De cada paciente se estudiaron variables demográficas, situación social, ingresos en los 3 años previos y días de hospitalización de año anterior al ingreso. Del ingreso índice se recogió diagnóstico, gravedad, complicaciones, estancia, tipo de ingreso, comorbilidad. Se valoró la capacidad funcional del paciente, el grado de dependencia en sus actividades diarias y la situación psicológica mediante test validados. Se determinó la existencia de reingreso a los 10, 28 días y 3 meses, definiéndolo como aquel motivado por el mismo diagnóstico principal. Se realizó estudio descriptivo de las variables y análisis univariado para establecer las que se relacionaban con el reingreso. Los factores pronósticos de reingreso se establecieron por análisis multivariado mediante regresión logística.

Resultados: La mediana de la edad fue 72 años y el $60,2 \%$ varones. El 75\% tenían sólo estudios primarios; el $40 \%$ eran solteros, separados o viudos; vivían solos el $12 \%$ y en asilos el $1,5 \%$. Los principales motivos de ingreso fueron: enfermedad pulmonar obstructiva crónica (EPOC), insuficiencia cardíaca congestiva (ICC), cardiopatía isquémica (C.Isq), arritmia cardíaca, enfermedad cerebrovascular aguda (ECVA), neumonía y asma; el ingreso fue de gravedad moderada en el 58,7\%. La media de la estancia fue de 3,5 días. La tasa de reingreso a los 10, 28 días y 3 meses fue, respectivamente, de 3,6, 9,7 y 13,3\%. El factor pronóstico independiente de reingreso a los 10 días fue el número de días ingresado el año previo (OR: 1,02, 95\% IC: 1,0-1,05). A los 28 días y a los 3 meses el único factor independiente fue el número de ingresos en los 3 años previos (OR: $1,71,95 \%$ IC: $1,32-2,22$ y OR: $1,62,95 \%$ IC: $1,28-2,05$ ).

Conclusiones: De los factores estudiados, los que pronostican reingreso hospitalario no planificado en pacientes ingresados en una Unidad de Corta Estancia no son modificables.

PALABRAS CLAVE: Calidad de asistencia. Eficiencia. Reingreso hospitalario. Unidad de Corta Estancia Médica. Regresión logística.

\section{ABSTRACT}

Aims: To determine the independent factors that predict unplanned hospital readmission in patients who are admitted to a Short-Stay Medi cal Unit (SSMU).

Design and field of the study: Prospective cohort. Sanitary area: 500000 inhabitants.

Subjects: Patients who survived to an hospital admission in the SSMU of our hospital ( $n=196, a=0.05, d=7)$.

Methods: We studied demographic variables, social situation, his tory of previous admissions to hospital, from each patient. From the index admission we included diagnosis, severity of the disease, compli cations, type of admission and co-morbility. Validated tests were done to measure funcional capability, grade of dependence and psicologic situa tion. After a three month interval readmission was determined if there had been an urgent admission to any hospital in a 10-day, 28-day or 3month interval after discharge caused by the same principal diagnosis. A descriptive analisis of the variables was made, and a univariate analisis to stablish their asociation to readmission. The predicting factors for readmission were calculated by means of logistic regression.

Results: The $60.2 \%$ were males, the median of the age was 72 years. $75 \%$ started or carried out only primary school; $40 \%$ were single, sepa rated or widowed; $12 \%$ lived alone and $1.5 \%$ in an institution. Principal diagnosis at the time of index admission were: chronic obstructive pul monary disease, congestive cardiac failure, ischemic heart disease, asth ma, pneumonia, cardiac arrythmia and acute cerebrovascular disease. Readmission rate at 10, 28 days and 3 months was 3.6\%, 9.7\% and $13.3 \%$, respectively. The independent predicting factor for readmission in the 10 days interval from discharge was the number of days of hospitalization in the last year (OR: 1.02, 95\% CI: 1.0-1.05). For readmission in a 28-day and 3-month interval from discharge, the only independent predicting factor was the number of admissions to any hospital in the last 3 years (OR: 1.71, 95\% CI: $1.32-2.22$ and OR: 1.62 , 95\% CI: $1.28-2.05$ respectively).

Conclusions: From the studied factors, those that predict unplanned hospital readmission of patients discharged from a Short-Stay Medical Unit cannot be modified.

KEY WORDS: Attendance quality. Efficiency. Hospital readmission. Short Stay Medical Unit. Logistic regression.

Diz-Lois Palomares MT, de la Iglesia Martínez F, Nicolás Miguel R, Pellicer Vázquez C, Ramos Polledo V, Diz-Lois Martínez F. Factores predictores de reingreso hospitalario no planificado en pacientes dados de alta de una Unidad de Corta Estancia Médica. An Med Interna (Madrid) 2002; 19: 221-225. 


\section{INTRODUCCIÓN}

El concepto de una buena asistencia sanitaria incluye calidad y eficacia optimizando el coste, lo que resume el término eficiencia. El índice de reingresos marca la evolución tras la asistencia hospitalaria por lo que es expresión de la calidad de la misma, siempre y cuando no se tenga en cuenta los ingresos programados ni los de otra causa no relacionada con el ingreso previo (1-3). La literatura afirma que cuanto más precoz es un reingreso, mayor es su indicación de una asistencia inadecuada en el ingreso previo4. Estos reingresos podrían deberse a un alta prematura, cuando el paciente aún precisaba atención hospitalaria. Más adelante en el tiempo, el reingreso es más probable que se deba a la propia evolución de la enfermedad, o a las características y comorbilidad de cada paciente. Los factores que se asocian a estos reingresos son un punto sobre el que se podría actuar para mejorar la eficiencia hospitalaria. Se han realizado estudios analizando distintas variables, en los que se concluye que el estadío de la enfermedad, la comorbilidad, la dependencia para las actividades de la vida diaria, los pacientes con EPOC o ICC y el número de ingresos previos se asocian a mayor número de reingresos (3,5-10).

Con el fin de mejorar la eficiencia se ha creado la Unidad de Corta Estancia Médica del Hospital Juan Canalejo de A Coruña, que funciona desde 1994. La UCEM admite un número aproximado de 3000 pacientes/año de características similares a las de plantas convencionales, con una estancia media de 3,7 días (11). El estudio de los reingresos en esta unidad tiene un especial interés por conocer los factores que los producen. También porque se ha apuntado la posibilidad de que la tendencia a disminuir la estancia hospitalaria ("alta precoz") podría llevar a un mayor número de reingresos $(10,12)$, si bien dicha tendencia no se ha confirmado en estudios realizados en plantas convencionales $(3,5,7)$.

Por ello se ha realizado un estudio para valorar los factores que determinan el reingreso hospitalario en pacientes que son dados de alta en la UCEM.

\section{MATERIAL Y MÉTODOS}

Se diseñó un estudio de cohorte prospectivo, sobre la población que ingresó y fue dada de alta en la UCEM del Hospital Juan Canalejo. Se recogió una muestra aleatoria de 196 pacientes dados de alta entre Noviembre de 1999 y Febrero de 2000. Se excluyó el período comprendido entre el 22 de Diciembre y el 7 de Enero, ya que en esta época los pacientes prefieren no estar ingresados y se adelantan altas hospitalarias, lo cual podría influir con un aumento en el número de reingresos posterior.

La muestra aleatoria fue recogida de pacientes consecutivos hasta un máximo diario, según el tiempo disponible. Se solicitó permiso oral para la realización de la entrevista y recogida de datos para este estudio. Un único autor (M.T. D.L.P.) entrevistó a cada paciente apuntando los datos demográficos: edad, sexo, dirección y teléfono, estado civil, con quién vivía, y estudios cursados. Se determinó el estatus funcional con el Indice de Karnofsky (13) (capacidad funcional) y el Indice de Barthel (14) (escala de dependencia), y se valoró el estatus psicológico con la escala de depresión-ansiedad de Goldberg (15). Se recogió el número de ingresos previos en los tres años anteriores, y los días de hospitalización en el año previo. Del ingreso índice se anotó el diagnóstico principal agrupado en nueve categorías (insuficiencia cardíaca, EPOC, C.isq, dolor torácico inespecífico, arritmia cardíaca, ECVA, neumonía, asma bronquial y otras), y su gravedad, definiéndose como leve en condiciones sin complicaciones o problemas de mínima severidad, moderada en problemas limitados a un órgano o sistema con riesgo aumentado de complicaciones y grave en afectación multiorgánica, sistémica generalizada o peor pronóstico. Se determinó la presencia o ausencia de complicaciones durante el ingreso. Se calcularon el índice de masa corporal, el índice de comorbilidad de Charlson (16) y el combinado de edad-Charlson (17); y, finalmente, se recogió la duración de la estancia en días del ingreso índice.

Al cabo de tres meses se investigó la presencia de reingreso en cualquier centro hospitalario, generalmente mediante contacto telefónico directo con el paciente o sus familiares, o bien mediante la revisión de la historia clínica. Se determinó el momento en que se produjo, el tipo de reingreso (urgente o programado), si el motivo coincidía con el diagnóstico principal del ingreso índice, y en caso de exitus, la causa del mismo. Se definió reingreso verdadero como aquel no programado motivado por el mismo diagnóstico principal.

Se realizó un estudio descriptivo de las variables anteriormente mencionadas, y un análisis univariado según la presencia o no de reingreso a los 10 días, 28 días ó 3 meses, determinando los factores que se asociaban a reingreso. Para este análisis se utilizó en variables cualitativas el test de Chi-cuadrado o test exacto de Fisher según procediese, y para variables cuantitativas el test de Mann-Whitney. Posteriormente con estas variables se realizó un estudio multivariado mediante regresión logística para determinar las variables independientes que se asocian a reingreso.

\section{RESULTADOS}

El $60 \%$ de los pacientes fueron varones y su mediana de edad de 72 años. Los demás datos socio-demográficos y la historia previa de ingresos están detallados en la tabla I. Los diagnósticos -motivo principal de ingreso fueron EPOC e ICC en la tercera parte de los casos. La mediana de la estancia hospitalaria fue de 3 días. La tabla II recoge otras características del ingreso índice.

El número de reingresos hospitalarios a los 10 días, 28 días y 3 meses fue de 7 pacientes $(3,6 \%), 19$ pacientes $(9,7 \%)$ y 26 pacientes $(13,3 \%)$, respectivamente (Fig. 1). Tras los 3 meses de seguimiento 16 pacientes fallecieron $(8,16 \%)$. El $75 \%$ de los éxitus se debió a la misma enfermedad principal que motivó el ingreso índice.

La tabla III resume los resultados del análisis univariado, tomando como variable dependiente los reingresos a los 10 días, 28 días y 3 meses. Se realizó el análisis multivariado controlando por las variables que presentaron significación estadística en el análisis previo. Considerando como variable dependiente el reingreso a los 10 días, la única variable independiente predictiva de reingreso fue los días de hospitalización en el año previo, con un riesgo relativo, $\mathrm{RR}=1,02,(95 \%$ IC: $1,0-1,05)$. Considerando como variable dependiente los reingresos a los 28 días y a los 3 meses, la única variable independiente predictiva de reingreso fue el número de ingresos en los 3 años anteriores, con un RR=1,71 (95\% IC: 1,32-2,22) y $\mathrm{RR}=1,62$ (95\% IC: $1,28-2,05)$ respectivamente. 
TABLA I

VARIABLES SO CIO-DEM O GRÁFICAS E HISTO RIA DE INGRESOS PREVIOS

\begin{tabular}{cccc}
\hline Mediana & $n(\%)$ & Rango & Percentiles \\
& & & $25-75$ \\
\hline
\end{tabular}

\section{Datos demográficos}

Edad, años

Sexo, varón

IMC, $\mathrm{kg} / \mathrm{m}^{2}$

72

$118(60,2)$

26,5

17-94

63-79

$17,3-44,9 \quad 23,4-29,3$

\section{Nivel Educacional}

No estudios

$23(11,7)$

Primarios incompletos

$65(33,2)$

$83(42,3)$

$20(10,2)$

$5(2,6)$

Secundarios

Universitarios

$22(11,2)$

$117(59,7)$

$48(24,5)$

$8(4,1)$

Otro

Convivencia habitual

$\begin{array}{lr}\text { Sólo } & 24(12,2) \\ \text { Familia } & 168(85,7) \\ \text { Asilo } & 3(1,5) \\ \text { Otro } & 1(0,5)\end{array}$

Número ingresos en los 31 años anteriores

Días de hospitalización 0 en el último año

n: número de casos. \% : porcentaje. IM C: índice de masa corporal.

\section{DISCUSIÓN}

El porcentaje de reingresos de nuestra cohorte se encuentra en el rango de encontrado por otros autores, de $4-5 \%$ a los 10 días, que varía desde el 6 al $20 \%$ a los 28 días, y alrededor del $20 \%$ a los 3 meses $(8,10,12)$. Observamos un mayor número de reingresos en los primeros días, objetivándose una disminución de la pendiente a partir del primer mes (Fig. 1). Ya otros autores detectan que de los reingresos relacionados a lo largo de 5 años (por la misma enfermedad o complicación de la misma), el $40 \%$ son tempranos (antes del primer mes)6. El aumento inicial se debe lógicamente en su mayoría a recaídas del mismo proceso. Debido a que el estudio ha sido realizado en los meses de invierno e inicio de la primavera, creemos que el porcentaje de reingresos encontrado es probablemente mayor que a lo largo de todo un año, pues en esta época son más frecuentes las exacerbaciones por viriasis, sobreinfecciones respiratorias, etc. Consideramos, por ello, que la época en la que se realizó el estudio constituye un sesgo, y que para conocer el porcentaje de reingresos real habría que ampliar el estudio a los meses de verano y otoño.

Otra limitación de nuestro estudio es la subjetividad de los observadores. Dicho sesgo quisimos minimizarlo decidiendo que únicamente un autor (MT D-L P) recogiese los datos de
TABLA II

\begin{tabular}{|c|c|c|c|}
\hline \multicolumn{4}{|c|}{ TABLA II } \\
\hline \multicolumn{4}{|c|}{ DESCRIPCIÓN DE VARIABLES DEL INGRESO ÍNDICE } \\
\hline Mediana & $n(\%)$ & Rango & $\begin{array}{c}\text { Percentiles } \\
25-75\end{array}$ \\
\hline Estancia, días & & $1-12$ & $2-4$ \\
\hline $\begin{array}{l}\text { Diagnóstico principal } \\
\text { EPOC } \\
\text { ICC } \\
\text { C. Isq } \\
\text { Dolor torácico inespecífico } \\
\text { Arritmia cardíaca } \\
\text { ECVA } \\
\text { Neumonía } \\
\text { Asma bronquial } \\
\text { Otros }\end{array}$ & $\begin{array}{r}35(17,9) \\
30(15,3) \\
16(8,2) \\
10(5,1) \\
14(7.1) \\
7(3,6) \\
12(6,1) \\
10(5,1) \\
62(31,6)\end{array}$ & & \\
\hline $\begin{array}{l}\text { Gravedad del ingreso } \\
\text { Leve } \\
\text { Moderada } \\
\text { Grave }\end{array}$ & $\begin{array}{r}25(12,8) \\
115(58,7) \\
56(28,6)\end{array}$ & & \\
\hline $\begin{array}{l}\text { Complicaciones del ingreso } \\
\text { Sí }\end{array}$ & $6(3,1)$ & & \\
\hline Índice de Charlson, puntos & & $0-9$ & $1-3$ \\
\hline Índice Edad-Charlson puntos 4 & & $0-12$ & $3-6$ \\
\hline $\begin{array}{l}\text { Índice de Karnofsky } \\
<40 \\
50 \\
60 \\
70 \\
80 \\
90 \\
100\end{array}$ & $\begin{array}{r}10(5,1) \\
11(5,6) \\
28(14,3) \\
42(21,4) \\
20(10,2) \\
24(12,2) \\
61(31,1)\end{array}$ & & \\
\hline $\begin{array}{l}\text { Índice de Barthel } \\
<20 \text {, dependiente total } \\
\text { 20-35, dependiente grave } \\
\text { 40-55, dependiente moderado } \\
\text { 60-95, dependiente leve } \\
\text { 100, independiente }\end{array}$ & $\begin{array}{r}7(3,6) \\
6(3) \\
4(2,1) \\
25(17,8) \\
144(73,5)\end{array}$ & & \\
\hline $\begin{array}{l}\text { Escala de ansiedad }(0-9) \\
0-1 \text { punto } \\
2-4 \text { puntos } \\
>5 \text { puntos }\end{array}$ & $\begin{array}{r}118(60,8) \\
60(31) \\
16(8,2)\end{array}$ & & \\
\hline $\begin{array}{l}\text { Escala de depresión (0-9) } \\
0-1 \text { punto } \\
\text { 2-4 puntos } \\
>5 \text { puntos }\end{array}$ & $\begin{array}{r}126(64,9) \\
45(23,2) \\
23(11,8)\end{array}$ & & \\
\hline
\end{tabular}

n: número de casos. \% : porcentaje. ICC insuficiencia cardíaca congestiva, EPO C: enfermedad pulmonar obstructiva crónica, C.Isq: cardiopatía isquémica, ECVA: enfermedad cerebrovascular aguda.

los pacientes y realizase los tests psicológicos y funcionales validados.

Los factores que se asocian a estos reingresos, ya sean a los 10 días, 28 días o 3 meses, son similares a lo señalado en la literatura, y son congruentes con el sentido común, pues son 


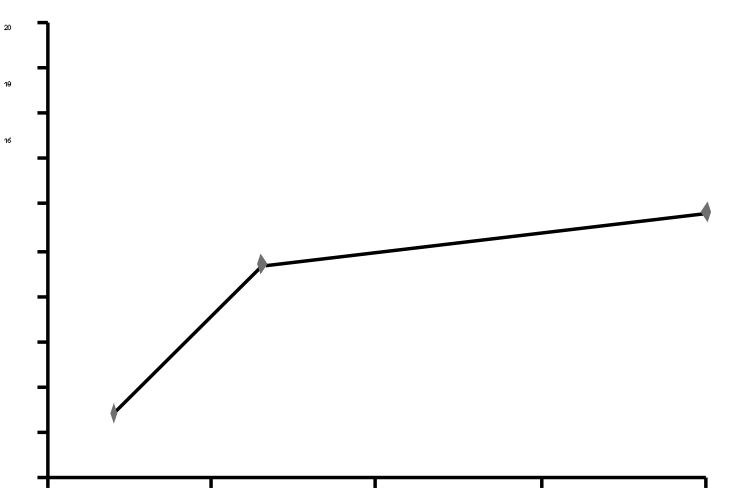

Fig. 1. Reingresos hospitalarios a los 10 días, 28 días y 3 meses, expresados en porcentaje del total de pacientes.

los enfermos con mayor comorbilidad y una larga historia clínica de ingresos previos los que más reingresan (5-7). No obstante, en nuestro estudio sólo son factores predictores de reingreso los días de hospitalización en el último año y el número de ingresos en los tres años anteriores. El hecho de que no encontrásemos otras variables significativas probablemente se deba a que nuestra muestra tiene un pequeño número de pacientes en el grupo de reingresos, lo que puede haber restado significación estadística a algunas variables.

La estancia durante el ingreso índice no influyó en el reingreso posterior, aunque la tendencia es que una mayor estancia se asocie positivamente al mismo $(3,5,7)$. El tipo de paciente es el que determina la probabilidad de reingreso. Los pacientes con enfermedades crónicas con constante necesidad de cuidados hospitalarios, peor recuperación y mayor convalecencia, son los que por un lado alargan la estancia y por otro aumentan los reingresos. Lo confirma el hecho de que pacientes con mayor índice de comorbilidad, y pacientes con enfermedades crónicas (ICC, EPOC) reingresen más, y a la vez, tiendan a presentar estancia hospitalarias más prolongadas, tanto en el nuestro como en otros estudios $(3,5,7)$.

Concluimos que los factores que predicen reingreso hospitalario verdadero en pacientes dados de alta de una Unidad de Corta Estancia Médica no son modificables, pues se trata de la historia de ingresos previos del paciente. No obstante, creemos que son necesarios nuevos estudios incluyendo a un mayor número de pacientes y a lo largo de todo el año para confirmar nuestros resultados.
TABLA II

RESULTADOS DE ANÁLISIS UNIVARIADO

\begin{tabular}{|c|c|c|c|}
\hline & \multicolumn{2}{|c|}{ Reingreso a los 10 días } & $\mathrm{P}$ \\
\hline & $\begin{array}{l}\text { Sí reingreso } \\
\text { (7 pac) }\end{array}$ & $\begin{array}{c}\text { No reingreso } \\
\text { (189 pac) }\end{array}$ & \\
\hline Edad, años & $79(53-83)$ & $72(63-79)$ & 0,30 \\
\hline Ingresos en 3 años anteriores & $2(1-3)$ & $1(0-2)$ & 0,038 \\
\hline $\begin{array}{l}\text { Días de hospitalización en el } \\
\text { último año }\end{array}$ & $8(0-21)$ & $0(0-7)$ & 0,058 \\
\hline Índice Edad-Charlson, puntos & $5(4-6)$ & $4(3-6)$ & 0,35 \\
\hline \multirow[t]{2}{*}{ Estancia, días } & $4(2-6)$ & $3(2-4)$ & 0,32 \\
\hline & $\begin{array}{l}\text { Reingres } \\
\text { Sír reingreso } \\
\text { (19 pac) }\end{array}$ & $\begin{array}{l}\text { los } 28 \text { días } \\
\text { No reingreso } \\
\text { (177 pac) }\end{array}$ & \\
\hline Edad, años & $79(68-84)$ & $72(63-79)$ & 0,055 \\
\hline Ingresos en 3 años anteriores & $2(1-4)$ & $1(0-2)$ & 0,000 \\
\hline $\begin{array}{l}\text { Días de hospitalización en el } \\
\text { último año }\end{array}$ & $12(0-21)$ & $0,(0-6)$ & 0,000 \\
\hline Indice Edad-Charlson, puntos & $6(4-7)$ & $4(3-6)$ & 0,043 \\
\hline Estancia, días & $4(3-5)$ & $3(2-4)$ & 0,164 \\
\hline \multirow[t]{2}{*}{$\begin{array}{l}\text { ICC } \\
\text { EPOC }\end{array}$} & $\begin{array}{l}31 \% \\
42 \%\end{array}$ & $\begin{array}{l}13 \% \\
15 \%\end{array}$ & 0,027 \\
\hline & $\begin{array}{l}\text { Reingres } \\
\text { Sír reingreso } \\
\text { (26 pac) }\end{array}$ & $\begin{array}{l}\text { los } 3 \text { meses } \\
\text { No reingreso } \\
\text { (170 pac) }\end{array}$ & \\
\hline Edad, años & $79(68-82)$ & $71(62-79)$ & 0,039 \\
\hline Ingresos en 3 años anteriores & $2(1-4)$ & $1(0-2)$ & 0,000 \\
\hline $\begin{array}{l}\text { Días de hospitalización en el } \\
\text { último año }\end{array}$ & $7(0-20)$ & $0(0-6)$ & 0,00 \\
\hline Índice Edad-Charlson, puntos & $5(4-6)$ & $4(3-6)$ & 0,048 \\
\hline Estancia, días & $4(2-5)$ & $3(2-4)$ & 0,08 \\
\hline $\begin{array}{l}\text { Diagnóstico en el ingreso-índice } \\
\text { ICC } \\
\text { EPOC }\end{array}$ & $\begin{array}{l}0,013 \\
27 \% \\
42 \%\end{array}$ & $\begin{array}{l}13 \% \\
14 \%\end{array}$ & \\
\hline
\end{tabular}

Resultados expresados como mediana (percentil 25-50) y \% : porcentaje.

\section{Bibliografía}

1. Milne R, Clarke A. Can readmission rates be used as an outcome indicator? BMJ 1990; 301: 1139-40.

2. Panzer RJ. Hospital readmissions and quality of care. Am J Med 1991; 90: 665-6.

3. Kossovsky MP, Perneger TV, Sarasin FP, Bolla F, Borst F, Gaspoz JM. Comparison between planned and unplanned readmissions to a department of internal medicine. J Clin Epidemiol 1999; 52: 151-6.

4. Sibbritt DW. Validation of a 28 day interval between discharge and readmission for emergency readmission rates. J Qual Clin Pract 1995; $15: 211-20$

5. Shipton S. Risk factors associated with multiple hospital readmissions Home Care Provid 1996; 1: 83-5.

6. Alonso Martínez JL, Llorente Diez B, Echegaray Agara M, Urbieta Echezarreta MA, González Arencibia C. Reingreso hospitalario en Medicina Interna. An Med Interna Madrid 2001; 18: 248-54.

7. Burns R, Nichols LO. Factors predicting readmission of older general medicine patients. J Gen Intern Med 1991; 6:389-93.

8. Phillips RS, Safran C, Cleary PD, Delbanco TL. Predicting emergency readmissions for patients discharged from the medical service of a teaching hospital. J. Gen Intern Med 1987; 2: 400-5. 
9. Gooding J, Jette AM. Hospital readmissions among the elderly. J Am Geriatr Soc 1985; 33: 595-601.

10. Williams EI, Fitton F. Factors affecting early unplanned readmission of elderly patients to hospital. BMJ 1988; 297: 784-7.

11. De la Iglesia Martínez F, Ramos Polledo V, Pellicer Vázquez C, Nicolás Miguel R, Diz-Lois Martínez F. La Unidad de Corta Estancia Médica (UCEM) de A Coruña: cumplimos cinco años. An Med Interna (Madrid) 2001; 18: 166.

12. Kwok T, Lau E, Woo J, Luk JK, Wong E, Sham A, et al Hospital readmission among older medical patients in Hong Kong. J R Coll Physicians Lond 1999; 33: 153-6.

13. Hutchinson TA, Boyd NF, Feinstein AR, Gouda A, Hollomby D, Rowat
B. Scientific problems in clinical scales, as demonstrated in the Karnofsky index of performance status. J Chronic Dis 1979; 32: 661-6.

14. Cid-Ruzafa J, Damian-Moreno J. Evaluación de la dependencia: escala de Barthel. Rev Esp Salud Pública 1997; 71: 127-37.

15. Gonçalves Estrella F, Fernández Camacho A. Síndromes ansioso-depresivos. En: Canals Lizano R y Marín Ibañez A, eds. Manual Práctico de Medicina General. Pentacrom S.L. Madrid 1998: 1113-27.

16. Charlson ME, Pompei P, Ales KL, MacKenzie CR. A new method of classifying prognostic comorbidity in longitudinal studies: development and validation. J Chronic Dis 1987; 40: 373-83.

17. Charlson M, Szatrowski TP, Peterson J, Gold J. Validation of a combined comorbidity index. J Clin Epidemiol 1994; 47: 1245-51. 